\title{
Status of the SCExAO instrument: recent technology upgrades and path to a system-level demonstrator for $\mathrm{PSI}$
}

\section{Lozi, Julien, Guyon, Olivier, Vievard, Sébastien, Sahoo, Ananya, Deo, Vincent, et al.}

Julien Lozi, Olivier Guyon, Sébastien Vievard, Ananya Sahoo, Vincent Deo, Nemanja Jovanovic, Barnaby Norris, Marc-Antoine Martinod, Ben Mazin, Alex Walter, Neelay Fruitwala, Sarah Steiger, Kristina Davis, Peter Tuthill, Tomoyuki Kudo, Hajime Kawahara, Takayuki Kotani, Michael Ireland, Theodoros Anagnos, Chrstian Schwab, Nick Cvetojevic, Elsa Huby, Sylvestre Lacour, Kevin Barjot, Tyler D. Groff, Jeffrey Chilcote, Jeremy Kasdin, Frantz Martinache, Romain Laugier, Mamadou N'Diaye, Justin Knight, Jared Males, Steven Bos, Frans Snik, David Doelman, Kelsey Miller, Eduardo Bendek, Ruslan Belikov, Eugene Pluzhnik, Thayne Currie, Masayuki Kuzuhara, Taichi Uyama, Jun Nishikawa, Naoshi Murakami, Jun Hashimoto, Yosuke Minowa, Christophe Clergeon, Yoshito Ono, Naruhisa Takato, Motohide Tamura, Hideki Takami, Masa Hayashi, "Status of the SCExAO instrument: recent technology upgrades and path to a system-level demonstrator for PSI," Proc. SPIE 11448, Adaptive Optics Systems VII, 114480N (13 December 2020); doi: $10.1117 / 12.2562832$ 


\section{Status of the SCExAO instrument: recent technology upgrades and path to a system-level demonstrator for PSI}

Julien Lozi ${ }^{\mathrm{a}}$, Olivier Guyon ${ }^{\mathrm{a}, \mathrm{b}, \mathrm{c}, \mathrm{d}}$, Sébastien Vievard ${ }^{\mathrm{a}, \mathrm{d}, \mathrm{e}}$, Ananya Sahoo $^{\mathrm{a}, \mathrm{f}}$, Vincent Deo ${ }^{\mathrm{a}}$, Nemanja Jovanovic $^{\mathrm{g}}$, Barnaby Norris ${ }^{\mathrm{h}}$, Marc-Antoine Martinod ${ }^{\mathrm{h}}$, Ben Mazin ${ }^{\mathrm{i}}$, Alex Walter ${ }^{\mathrm{i}}$, Neelay Fruitwala ${ }^{\mathrm{i}}$, Sarah Steiger ${ }^{\mathrm{i}}$, Kristina Davis ${ }^{\mathrm{i}}$, Peter Tuthill ${ }^{\text {, }}$, Tomoyuki Kudo ${ }^{\mathrm{a}, \mathrm{d}}$, Hajime Kawahara $^{j}$, Takayuki Kotani ${ }^{\mathrm{k}, \mathrm{d}}$, Michael Ireland ${ }^{\mathrm{l}}$, Theodoros Anagnos ${ }^{\mathrm{m}}$, Chrstian Schwab $^{\mathrm{m}}$,

Nick Cvetojevic $^{\mathrm{n}}$, Elsa Huby ${ }^{\mathrm{e}}$, Sylvestre Lacour ${ }^{\mathrm{e}}$, Kevin Barjot ${ }^{\mathrm{e}}$, Tyler D. Groffo ${ }^{\mathrm{o}}$, Jeffrey Chilcote $^{\mathrm{p}}$, Jeremy Kasdin ${ }^{\mathrm{q}}$, Frantz Martinache ${ }^{\mathrm{n}}$, Romain Laugier ${ }^{\mathrm{n}}$, Mamadou N'Diaye ${ }^{\mathrm{n}}$, Justin Knight $^{\mathrm{b}}$, Jared Males ${ }^{\mathrm{b}}$, Steven Bos ${ }^{\mathrm{r}}$, Frans Snik ${ }^{\mathrm{r}}$, Davis Doelman ${ }^{\mathrm{r}}$, Kelsey Miller ${ }^{\mathrm{r}}$, Eduardo Bendek $^{\mathrm{s}}$, Ruslan Belikov ${ }^{\mathrm{t}}$, Eugene Pluzhnik ${ }^{\mathrm{t}}$, Thayne Currie ${ }^{\mathrm{t}}$, Masayuki Kuzuhara ${ }^{\mathrm{k}}$, Taichi Uyama $^{\mathrm{g}}$, Jun Nishikawa ${ }^{\mathrm{k}}$, Naoshi Murakami ${ }^{\mathrm{u}}$, Jun Hashimoto ${ }^{\mathrm{k}}$, Yosuke Minowa ${ }^{\mathrm{a}, \mathrm{k}}$, Christophe Clergeon $^{\mathrm{a}}$, Yoshito Ono ${ }^{\mathrm{a}}$, Naruhisa Takato ${ }^{\mathrm{a}, \mathrm{k}}$, Motohide Tamura ${ }^{\mathrm{k}, \mathrm{d}, \mathrm{j}}$, Hideki Takami ${ }^{\mathrm{k}}$, and Masa Hayashi ${ }^{\mathrm{k}}$

aSubaru Telescope, National Astronomical Observatory of Japan, National Institutes of Natural Sciences (NINS), 650 North A'ohōkū Place, Hilo, HI, 96720, U.S.A.

bSteward Observatory, University of Arizona, Tucson, AZ, 85721, U.S.A.

${ }^{c}$ College of Optical Sciences, University of Arizona, Tucson, AZ 85721, U.S.A.

dAstrobiology Center of NINS, 2-21-1, Osawa, Mitaka, Tokyo, 181-8588, Japan

${ }^{\text {e} O b s e r v a t o i r e ~ d e ~ P a r i s, ~ L E S I A, ~} 5$ Place Jules Janssen, 92190 Meudon, France

fSokendai, Graduate University for Advanced Studies, Kanagawa Prefecture, Miura District, Hayama, Shonankokusaimura, 240-0193, Japan

${ }^{\text {g} C a l i f o r n i a ~ I n s t i t u t e ~ o f ~ T e c h n o l o g y, ~} 1200$ E California Blvd, Pasadena, CA 91125, U.S.A.

hSydney Institute for Astronomy, Institute for Photonics and Optical Science, School of

Physics, University of Sydney, NSW 2006, Australia

${ }^{\mathrm{i} U n i v e r s i t y ~ o f ~ C a l i f o r n i a ~ S a n t a ~ B a r b a r a, ~ S a n t a ~ B a r b a r a, ~ C A ~ 93106, ~ U . S . A . ~}$

jUniversity of Tokyo, 7 Chome-3-1 Hongo, Bunkyo City, Tokyo 113-8654, Japan

kNational Astronomical Observatory of Japan, National Institutes of Natural Sciences (NINS),

2 Chome-21-1 Osawa, Mitaka, Tokyo 181-0015, Japan

${ }^{1}$ The Australian National University, Canberra ACT 0200, Australia

macquarie University, Balaclava Rd, Macquarie Park NSW 2109, Australia

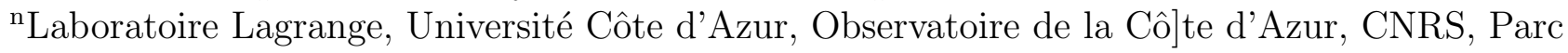

Valrose, Bât. H. FIZEAU, 06108 Nice, France

${ }^{\circ}$ Goddard Space Flight Center, 8800 Greenbelt Rd, Greenbelt, MD 20771, U.S.A

pUniveristy of Notre Dame, Notre Dame, IN 46556, U.S.A.

qPrinceton University, Princeton, NJ 08544, U.S.A.

${ }^{\mathrm{r}}$ Leiden Observatory, Leiden University, P.O. Box 9513, 2300 RA Leiden, The Netherlands

s Jet Propulsion Laboratory, 4800 Oak Grove Dr, Pasadena, CA 91109

${ }^{t}$ NASA Ames Research Center, Moffett Blvd, Mountain View, CA 94035, U.S.A.

uHokkaido University, 5 Chome Kita 8 Jonishi, Kita Ward, Sapporo, Hokkaido 060-0808, Japan

Further author information: (Send correspondence to J.L.)

J.L.: E-mail: lozi@naoj.org, Telephone: 18089345949

Adaptive Optics Systems VII, edited by Laura Schreiber, Dirk Schmidt, Elise Vernet, Proc. of SPIE Vol. 11448, 114480N · C 2020 SPIE · CCC code: 0277-786X/20/\$21 - doi: 10.1117/12.2562832 


\begin{abstract}
The Subaru Coronagraphic Extreme Adaptive Optics (SCExAO) instrument is a high-contrast imaging system installed at the 8-m Subaru Telescope on Maunakea, Hawaii. Due to its unique evolving design, SCExAO is both an instrument open for use by the international scientific community, and a testbed validating new technologies, which are critical to future high-contrast imagers on Giant Segmented Mirror Telescopes (GSMTs). Through multiple international collaborations over the years, SCExAO was able to test the most advanced technologies in wavefront sensors, real-time control with GPUs, low-noise high frame rate detectors in the visible and infrared, starlight suppression techniques or photonics technologies. Tools and interfaces were put in place to encourage collaborators to implement their own hardware and algorithms, and test them on-site or remotely, in laboratory conditions or on-sky. We are now commissioning broadband coronagraphs, the Microwave Kinetic Inductance Detector (MKID) Exoplanet Camera (MEC) for high-speed speckle control, as well as a C-RED ONE camera for both polarization differential imaging and IR wavefront sensing. New wavefront control algorithms are also being tested, such as predictive control, multi-camera machine learning sensor fusion, and focal plane wavefront control. We present the status of the SCExAO instrument, with an emphasis on current collaborations and recent technology demonstrations. We also describe upgrades planned for the next few years, which will evolve SCExAO - and the whole suite of instruments on the IR Nasmyth platform of the Subaru Telescope - to become a system-level demonstrator of the Planetary Systems Imager (PSI), the high-contrast instrument for the Thirty Meter Telescope (TMT).
\end{abstract}

Keywords: Manuscript format, template, SPIE Proceedings, LaTeX

\title{
1. INTRODUCTION
}

For the past 11 years, the Subaru Coronagraphic Extreme Adaptive Optics (SCExAO) ${ }^{1}$ evolved into a modular high-contrast imaging instrument and testbed, capable of developing and testing new technologies, hardware and algorithms, in laboratory conditions and on-sky. This unique capabilities is crucial to test the necessary technologies that future high-contrast instruments for Giant Segmented Mirror Telescopes (GSMTs) will require. One such instrument, the Planetary Systems Imager (PSI $)^{2}$ developed for the Thirty Meter Telescope (TMT), ${ }^{3}$ will be composed of two main parts, PSI-blue and PSI-red. PSI-red will provide the first level of correction with a common deformable mirror (DM) and analyze the light between 2 and $5 \mu \mathrm{m}$, while the more challenging PSI-blue will provide Extreme Adaptive Optics (ExAO) performance between 0.6 and $1.8 \mu \mathrm{m}$ with a second DM, and analyze the light at these wavelengths. SCExAO is already close to the PSI-blue configuration, but future major hardware changes inside and in front of SCExAO will change the instrument configuration at the Nasmyth platform of Subaru into a combination of PSI-blue and red.

In this paper, we present the current status of SCExAO, especially the main components similar to other high-contrast imagers, then we describe the experimental modules that are unique to the instrument, and what makes SCExAO a great platform for collaborations. Finally, we detail the major upgrades planned for SCExAO and AO188, that will bring the instrument closer to a system-level demonstrator of PSI.

\section{2. "CLASSICAL" MODULES OF HIGH-CONTRAST IMAGING MODES OF SCExAO}

Similarly to SPHERE ${ }^{4}$ and GPI, ${ }^{5}$ SCExAO has the key components of high-contrast imagers and the main one is the extreme AO loop. While SPHERE has a single ExAO stage and GPI has a two-stage correction with a woofer and a tweeter DM, SCExAO relies on an independent first stage correction from Subaru Telescope's facility adaptive optics AO188, a 188-element AO correcting the atmospheric turbulence to typical Strehl ratios of 20 to $40 \%{ }^{6}$. On top of that, SCExAO performs the second level of correction, using a 2000-actuator deformable mirror (DM) to achieve ExAO performance (Strehl ratios $>80 \%)^{1,7}$

Figure 1 shows the effect of the first level of correction by AO188, and the extreme AO correction by SCExAO. With SCExAO, the speckle halo around the PSF is more stable, and at a lower level, allowing for better sensitivity when looking for companions and disks. The ExAO loop uses the visible light between 800 and $900 \mathrm{~nm}$, while the infrared light $(>950 \mathrm{~nm})$ is mostly used for science. The wavefront sensing is performed by a 


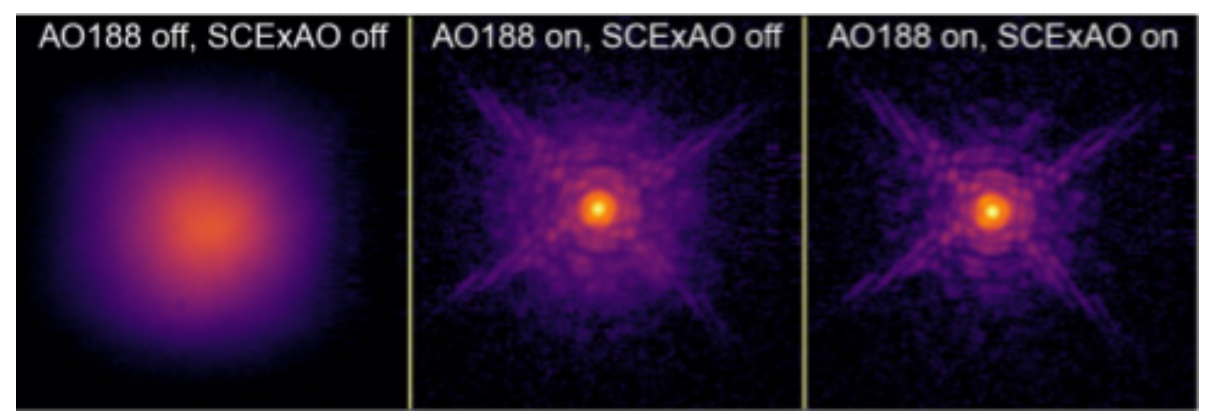

Figure 1. On-sky PSF in seeing limited mode (left), after the first stage of correction by AO188 (middle), after extreme AO correction (right). With SCExAO, the PSF is more stable, and the speckle halo surrounding the core is fainter.

modulated Pyramid Wavefront Sensor (PyWFS) that combines a double roof prism pyramid optics and a First Light Imaging OCAM2K EMCCD camera. ${ }^{7}$

In the infrared path, the starlight is masked by a coronagraph, in most cases a classical Lyot coronagraph with an Inner Working Angle (IWA) of 113 mas. The light rejected by the Lyot stop can be directed towards a low-order wavefront sensor (LOWFS) that removes dynamic low-order aberrations seen by the coronagraph. ${ }^{8}$ The Lyot coronagraph is currently our most popular coronagraph, since it is the most broadband option we have.

Most of the science in NIR is performed by the Integral Field Spectrograph (IFS) CHARIS, ${ }^{9}$ using J-, Hand K-band $(1.1$ to $2.4 \mu \mathrm{m})$. Figure 2 presents a few results obtained with SCExAO and CHARIS. Typical

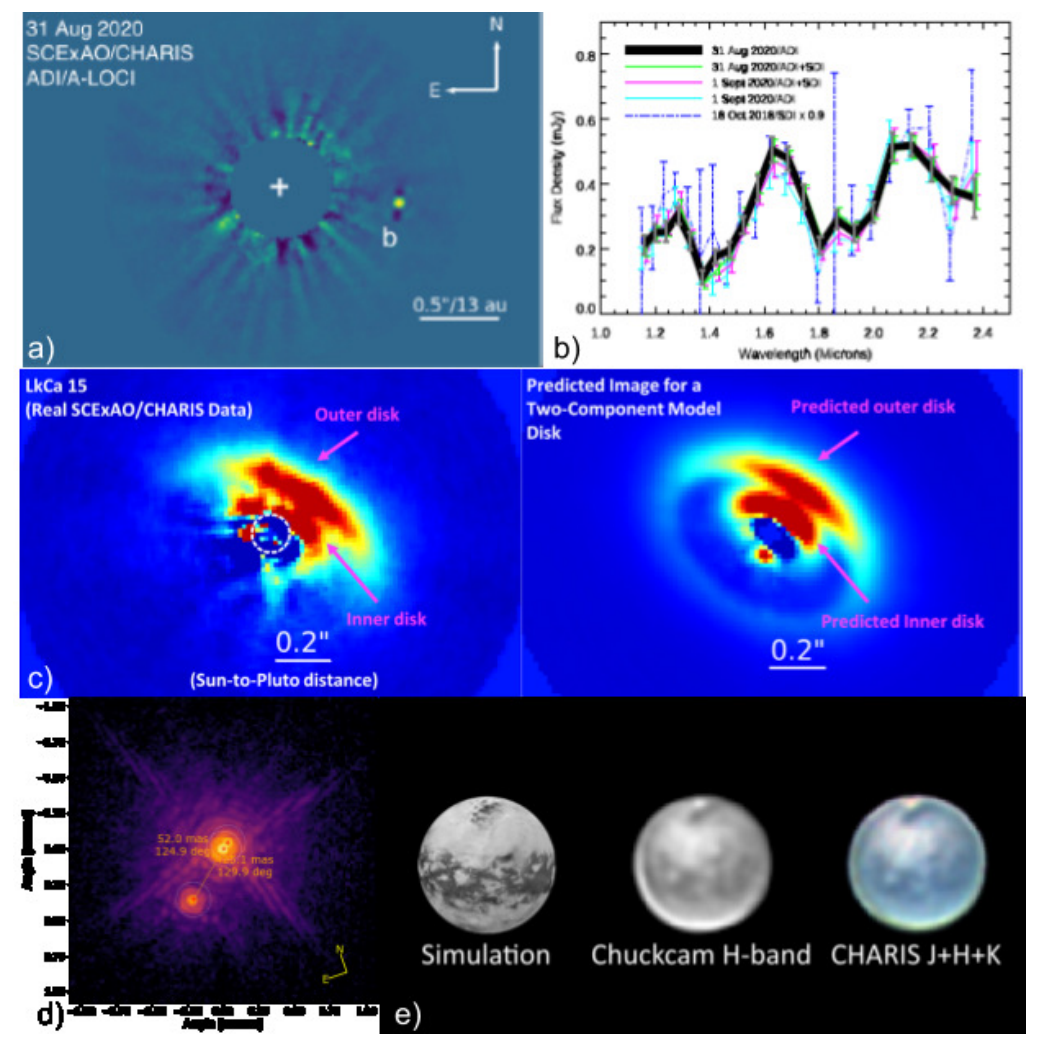

Figure 2. Examples of science images taken with SCExAO: a) Recent discovery of a low-mass brown dwarf companion around HD 33632 A, b) Spectrum of that companion taken with the broadband mode of CHARIS, c) protoplanetary disk around LkCa 15 taken with CHARIS compared to a simulation of that same disk, d) triple star system discovered by CHARIS when a binary was expected, and e) Observation of Titan with CHARIS and the internal IR camera of SCExAO, and comparison with current maps. 

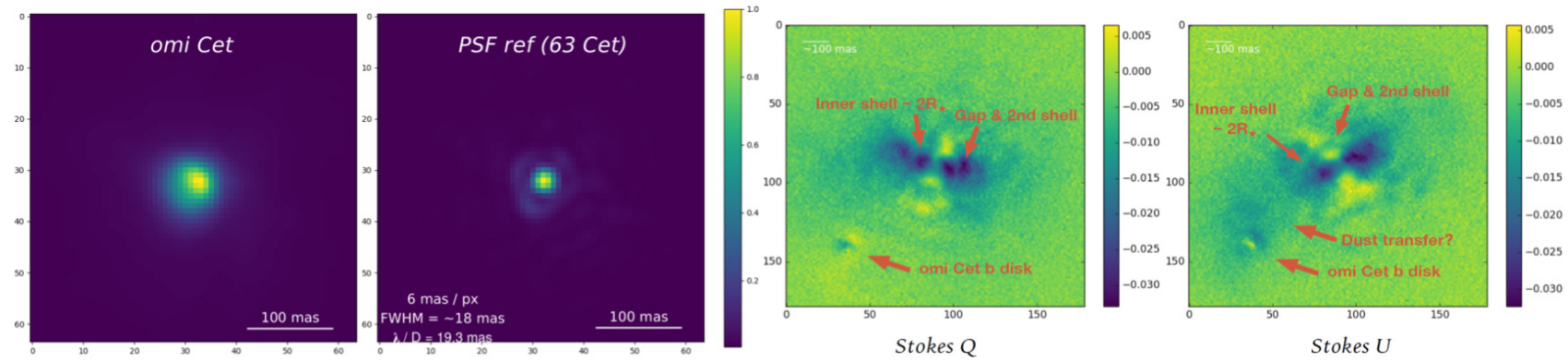

Figure 3. Examples of results obtained with VAMPIRES: The post-AGB star Mira (o Cet) is resolved by VAMPIRES, compared to a reference star. In polarized light, shells of dust are visible around Mira A, while a disk is also visible around its companion Mira B.

targets are low-mass companions such as HD 33632 Ab (Fig. 2 a)) and perform some spectroscopic analysis using CHARIS (Fig. 2 b)). ${ }^{10}$ We also routinely observe protoplanetary and debris disks such as the one around LkCA 15 (Fig. 2 d)). ${ }^{11}$ In that case, careful modelizations show that the candidate planets previously observed were only parts of an inner disk. Other cameras can also be used in parallel with CHARIS, like SCExAO's fast internal NIR camera from y- to H-band $(0.95$ to $1.7 \mu \mathrm{m})$, used for example to acquire this image of a triple system discovered when a binary was expected (Fig. 2 d)), or this image of Titan (Fig. 2 e)) compared to known maps created from Cassini data.

One of the most successful features of SPHERE and GPI are their capacity to measure polarization, mostly to image disks. SCExAO was equipped from the beginning with VAMPIRES, a module doing polarization differential imaging (PDI) in visible using a pair of fast EMCCD cameras, and a Ferroelectric Liquid Crystal (FLC) capable of modulating the polarization synchronized with the frame rate of the cameras. VAMPIRES is also equipped with some aperture masks to increase slightly the resolution for some targets. Figure 3 presents some results obtained with VAMPIRES on the post-AGB star Mira.

Recently, new PDI modes were added on the NIR path. A spectro-polarimetric mode using CHARIS, created by adding a Wollaston in front of the instrument, allows to keep the spectroscopic capability of the instrument, while separating the two polarization states. The field-of-view of this mode is thus reduced by a factor 2 , to

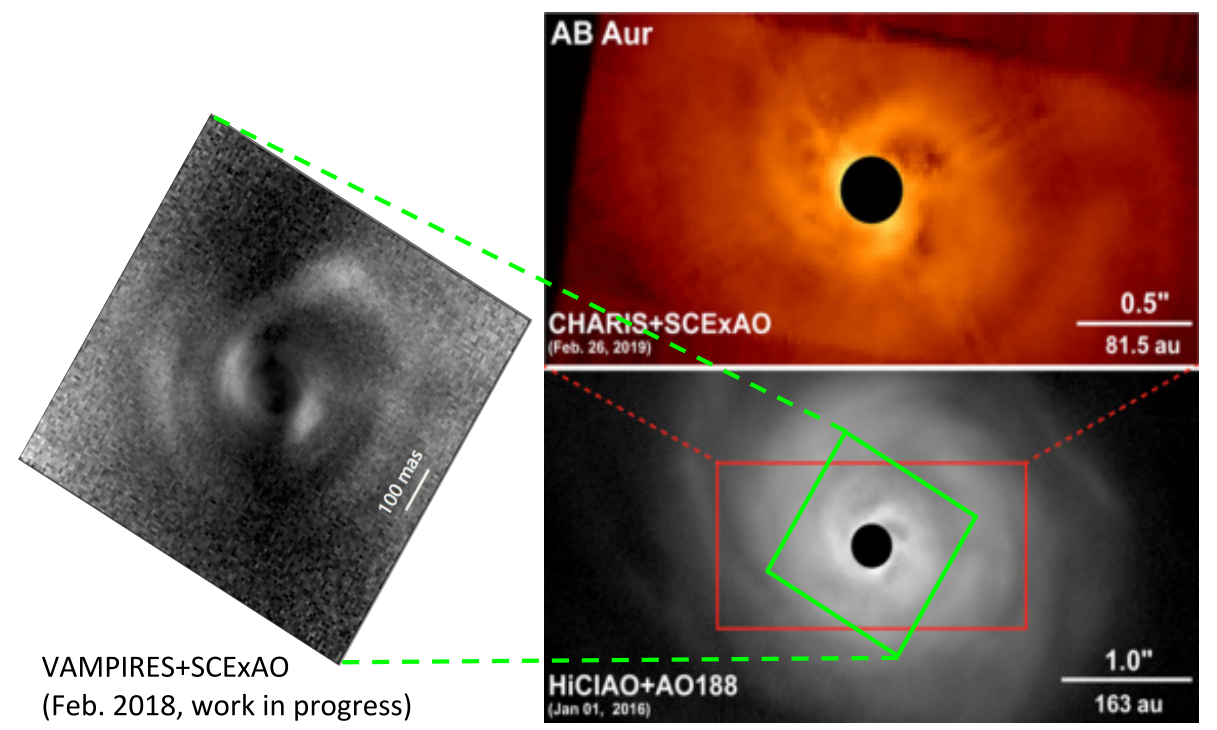

Figure 4. Comparison of the polarimetric intensity images between old HiCIAO observations (2016), and CHARIS observations (2019). In the CHARIS images, the wavelengths were collapsed. The CHARIS image shows a slightly better resolution and inner working angle. In comparison, we also present a VAMPIRES image (work in progress) of the central region. Residual spider diffraction is still visible in the CHARIS image, probably due to uncorrected distortion. 
$1 " \mathrm{x} 2$ ". This mode is now available for Open-Use observations.

Figure 4 is an example of polarization imaging of the swirling disk of dust around $\mathrm{AB}$ Aur ${ }^{12}$ in visible with VAMPIRES, and comparing the old PDI mode of HiCIAO and the new one with CHARIS.

In addition, a second NIR polarimetric mode is currently in progress, using a fast IR detector, the C-RED ONE camera, and a synchronized FLC similarly to VAMPIRES. This mode will be available soon (see Lozi et al. from this conference).

Finally, SCExAO was also equipped with a single-mode fiber injection module, linked to the high-resolution spectrograph IRD. ${ }^{13}$ This module, named REACH, combines the high-contrast capability of SCExAO with the high spectral resolution of IRD. The fiber used for the injection has multiple cores, allowing for a core to sample the light from a companion (exoplanet, brown dwarf) and other cores to sample only residual light from the central star.

Despite having all the components of other high-contrast imagers, SCExAO is not planing on doing the same type of surveys yet. This is mostly because the instrument is constantly evolving, with new hardware and algorithms. The modular design of the instrument allows to test new technologies, necessary for future high-contrast imagers, therefore compromising on the stability necessary for long surveys.

\section{AN INFRASTRUCTURE FOR EXPERIMENTAL WORK}

\subsection{A Modular Design}

During the past 11 years of operation, SCExAO underwent through several major redesign, to accommodate new modules and other hardware upgrades. The instrument is mostly based on collaborative efforts with multiple teams around the world, building its various components. The instrument's hardware and software evolved to allow for quick prototyping and testing of new technologies, in laboratory conditions and on-sky. The optical path is equipped with several wheels allowing to add optics and masks in the various pupil planes and focal planes along the way, as well as dichroics, beam-splitters and pickoff mirrors, distributing the light to the different modules. A calibration source can be inserted in the focal plane instead of the beam coming from AO188, for laboratory testing even when the instrument is not installed behind the telescope.

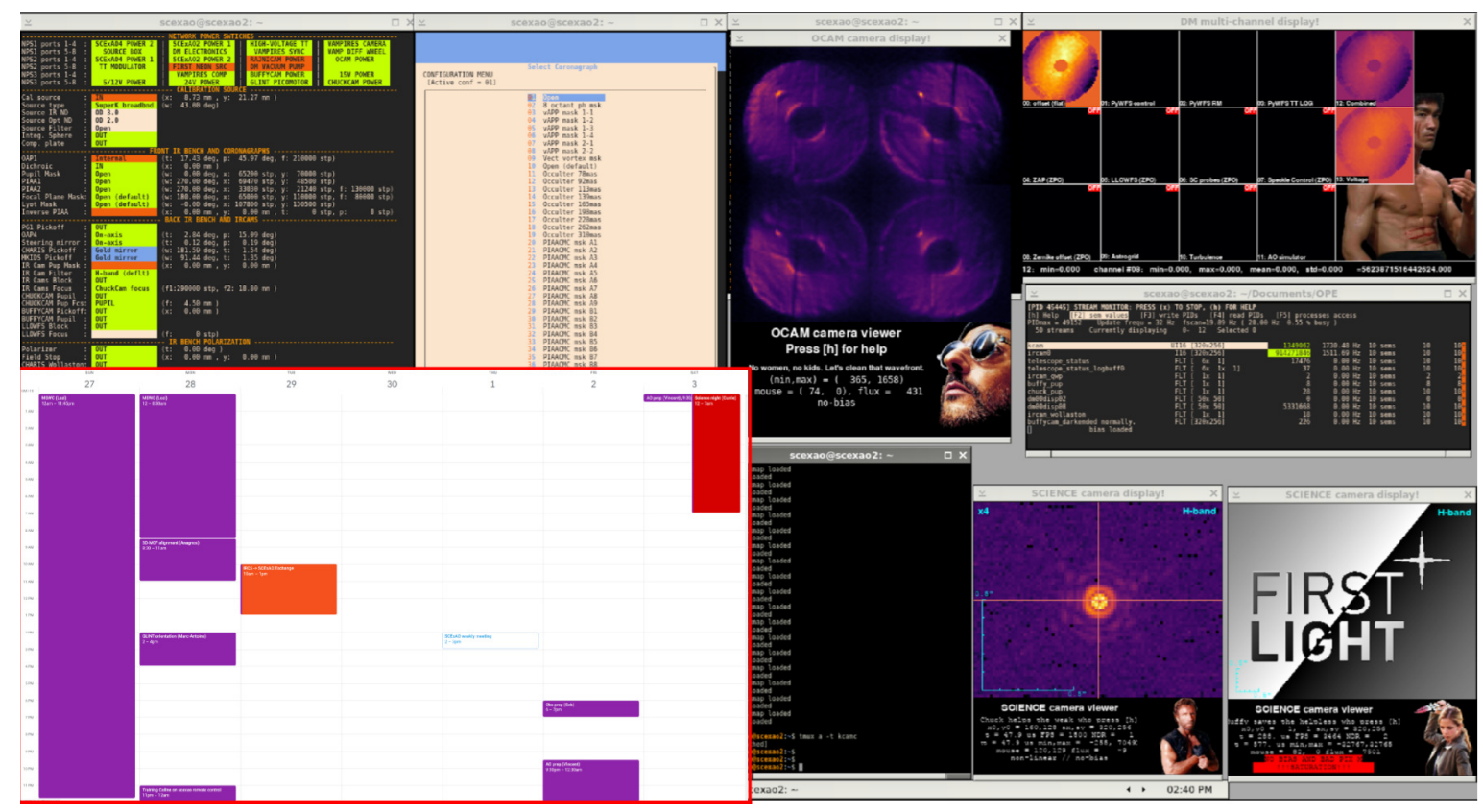

Figure 5. main VNC window controlling the hardware of SCExAO, with a status of the various motors, control of the coronagraphs, and display of the different cameras and the DM. In insert (bottom left) is an example of the Google calendar used to schedule laboratory testing. 
The instrument is available almost $24 / 7$ for collaborators to use - minus observing nights and when the instrument is down for maintenance-, after we provide a VPN access and some training on the different necessary procedures. The team in Hilo, Hawaii is usually there ready to assist, especially if hardware changes and/or alignments are required at the telescope. Figure 5 presents an example of VNC terminal used to control the hardware on the instrument, as well as the Google Calendar used to coordinate laboratory testing, on-sky observations and maintenance. From this VNC terminal, we can display the status of all the moving parts of the instrument, control the various coronagraph, control and display the various cameras and wavefront sensors, and display the status of the DM.

The software architecture is designed to be flexible, modular and open-source (see https://github.com/ scexao-org). It is designed to benefit from CPUs and GPUs available, and to manage various data flows from various sources (wavefront sensors, cameras, other telemetry) and different frame rates. The Modular Image processing Library toolkit (MILK) manages the data structures for basic input-output functionalities, data processing, etc., while the Compute and Control for Adaptive Optics (CACAO) package manages the real-time control (AO loops, predictive control, etc. $)^{14}$

The streams used are shared memory data structures, that can be directly accessed from a RAM disk, and read and written by as many processes as needed. The collisions are then avoided by using semaphores. Thanks to this architecture, it is easy for any user to plug and play their own codes, usually in Python or C (although other languages are possible), and not care about the hardware interfaces from the cameras are to the DM.

\subsection{Overview of the Current Collaborations}

On the hardware side, the modularity of the instrument allows us to test a variety of small IWA coronagraphs, such as the vector vortex coronagraph, the 8-octant phase mask coronagraph (see Nishikawa et al. from this conference), or the phase-induced amplitude apodization complex mask coronagraph. ${ }^{15}$ Pupil masks changing the diffraction pattern for high-contrast imaging are also tested, such as the shaped pupil ${ }^{16}$ or the vector Apodizing Phase Plate (vAPP). ${ }^{17}$

We are also investigating ways of improving the astrometric and photometric calibration of the science images, when the coronagraph is in place. While we currently use a temporally modulated speckle grid created by the deformable mirror, we showed that adding a spatial modulation increased the precision of the calibration. ${ }^{18}$ Other techniques are also investigated, such as an incoherent speckle grid created by a polarizing phase plate (see Bos et al. from this conference).

In addition to this, SCExAO is equipped with several experimental interferometric and fiber injection modules, that we are envisioning as science modules, but also as part of the wavefront sensing inputs:

- FIRST, a visible non-redundant mapping interferometer with spectroscopic capability, ${ }^{19}$

- GLINT, a NIR photonic nulling interferometer (see Martinod et al. from this conference),

- RHEA, a visible $3 \times 3$ single-mode fiber injection IFS connected to a high-resolution spectrograph ${ }^{20}$ (see Anagnos et al. from this conference),

- A NIR version of RHEA, where we explore using 3D-printed lenslets on a multi-core fiber, as a demonstrator for the visible one,

- an innovative fiber injected low-order wavefront sensor using a photonic lantern (see Norris et al. from this conference).

Besides hardware modules, the most fruitful collaborations on the instrument are on innovative wavefront control algorithms, especially using focal plane images. For the past few years, we tested several algorithms aimed at measuring and correcting quasi-static non-common path aberrations, low-order errors, or low-wind/island effect. Since SCExAO is only equipped with one DM, the commands from the other correction loops are applied by offsetting the reference of the ExAO loop.

The algorithms tested so far are: 

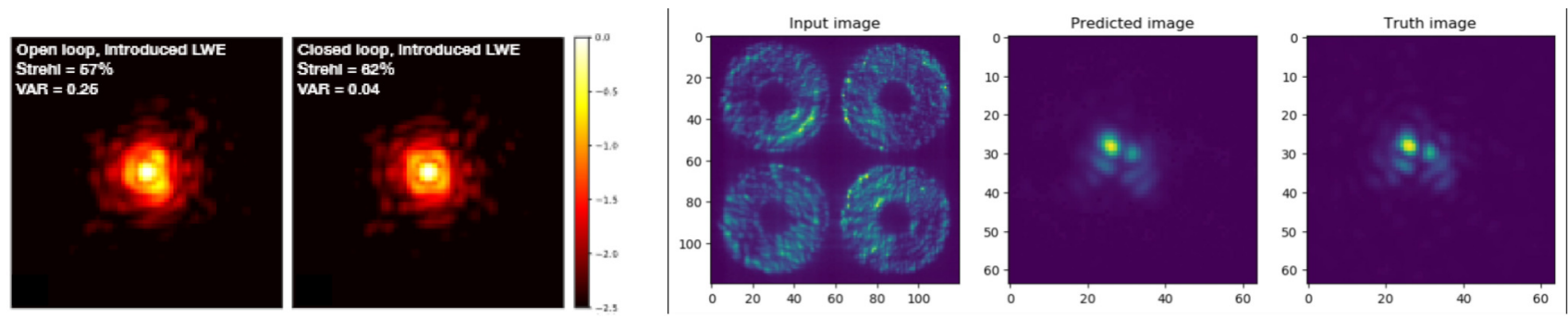

Figure 6. Example of focal plane wavefront control using the Fast \& Furious algorithm ${ }^{21}$ (left), and focal plane image reconstruction from PyWFS data using a neural network (right).

- Zernike Asymmetric Pupil (ZAP) WFS: a phase retrieval algorithm using an asymmetric pupil mask, ${ }^{22}$

- phase diversity algorithms (Linearized Analytic phase diversity, Mono-plan phase diversity, Fast \& Furious, ${ }^{21}$ see example in Fig. 6),

- PSF Reconstruction from PyWFS images, using a neural network (see Fig. 6),

- Direct Reinforcement Wavefront Heuristic Optimization (DR WHO): An algorithm based on reinforcement learning.

In addition to the correction of low-order aberrations, we developed several speckle control techniques like speckle nulling, ${ }^{23}$ necessary to reach deeper contrasts by correcting high-order aberrations unseen by the PyWFS. Their on-sky implementation was proven quite difficult, as they tend to require a very stable wavefront correction, and a good calibration of the pyramid WFS.

On-sky implementation of speckle nulling requires fast IR detectors, such as Institute for Astronomy's SAPHIRA camera, ${ }^{24}$ the C-RED ONE camera, or the Microwave Kinetic Inductance Detector (MKID) Exo-
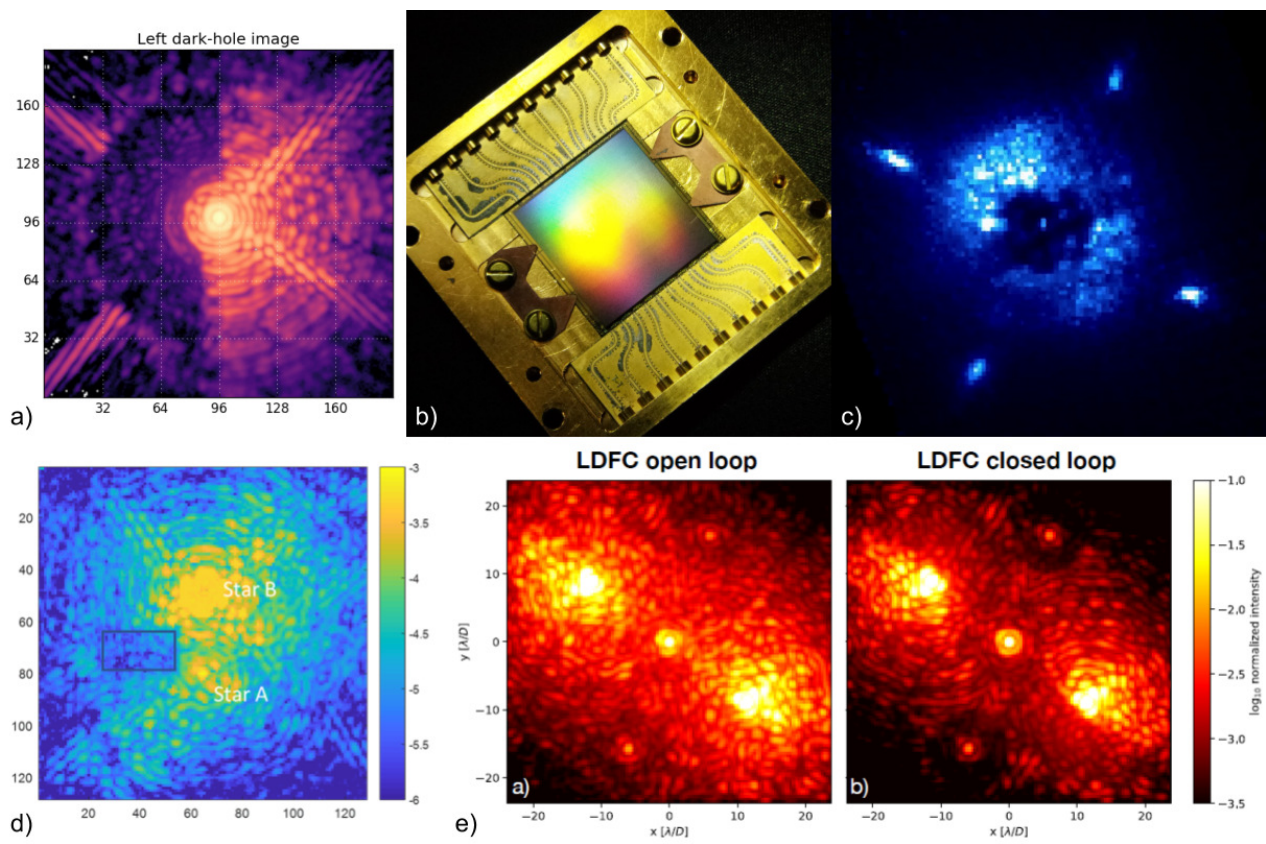

Figure 7. Testing of speckle control algorithms: a) lab demonstration of speckle nulling on SCExAO, b) 20,000-pixel MKID detector of MEC, c) preliminary result of MEC on-sky, the speckle halo can be seen around the coronagraph, as well as a companion on the right, d) lab demonstration of multi-star wavefront control on a simulated binary system, e) on-sky demonstration of the dark hole stabilization technique LDFC. 
planet Camera (MEC, see Fig. $7 \mathrm{~b}$ ) and c)), which is a photon counting camera that can measure the energy of the incoming photons.

We are also testing less conventional approaches, such as the multi-star wavefront control (MSWC, see Fig. 7 d)), a speckle nulling algorithm used for binary systems (see Belikov et al. from this conference). Finally, we are demonstrating algorithms to stabilize the dark hole over longer periods of time, notably by measuring the disturbances using the opposite bright field. This technique is called Linear Dark Field Control (LDFC). ${ }^{25}$

All these collaborations are solving new challenges that will face future high-contrast imagers, and therefore are essential if we ever want to reach the contrast necessary to detect earth-like planets with GSMTs.

\section{STEPS TO GET CLOSER TO THE TMT-PSI CONFIGURATION}

With 8-m telescopes such as Subaru, it is impossible to image Earth-like planets in the habitable zone of stars. However, the new generation of GSMTs provides the necessary resolution to probe close to a significant number of M-type stars. But to reach the necessary contrast to image Earth-like planets around these stars, a significant improvement in wavefront control needs to be reached.

One instrument that will target Earth-like planets in the habitable zone of M-type stars is the Planetary Systems Imager (PSI), planned to be installed on the Thirty Meter Telescope (TMT). The design of PSI, presented in Fig. 8, is composed of two main parts: PSI-red and PSI-blue. PSI-red will analyze wavelengths between 2 and $5 \mu \mathrm{m}$, while PSI-blue will analyze wavelengths between 0.6 and $2 \mu \mathrm{m}$. A third port can potentially send light over $5 \mu \mathrm{m}$ to a $10 \mu \mathrm{m}$ imager, or a mid-IR IFS. An IR wavefront sensor would send the first step of correction to a woofer DM, common to all the modes. The NIR science light would go either to a low- to mid-resolution IFS, or a single-mode fiber injected high-resolution spectrograph. PSI-blue would have its own twitter DM driven by a visible WFS, and the science light would also go to either an IFS or a single-mode fiber injected high-resolution spectrograph.

SCExAO is close to the PSI-blue configuration, although both stages of wavefront control are currently only performed in visible. Over the next few years, a series of major upgrades in AO188 will bring the instrument configuration on the Nasmyth IR platform of Subaru to something closer to PSI-blue and red.

AO188 recently received an upgrade of its real-time computer with faster hardware, and the CACAO infrastructure to run the control loop. ${ }^{26}$ This upgrade will allow to operate SCExAO and AO188 in a woofer/tweeter configuration. We can now get real-time telemetry of the AO188 wavefront sensor, and perform offsets between SCExAO and AO188.

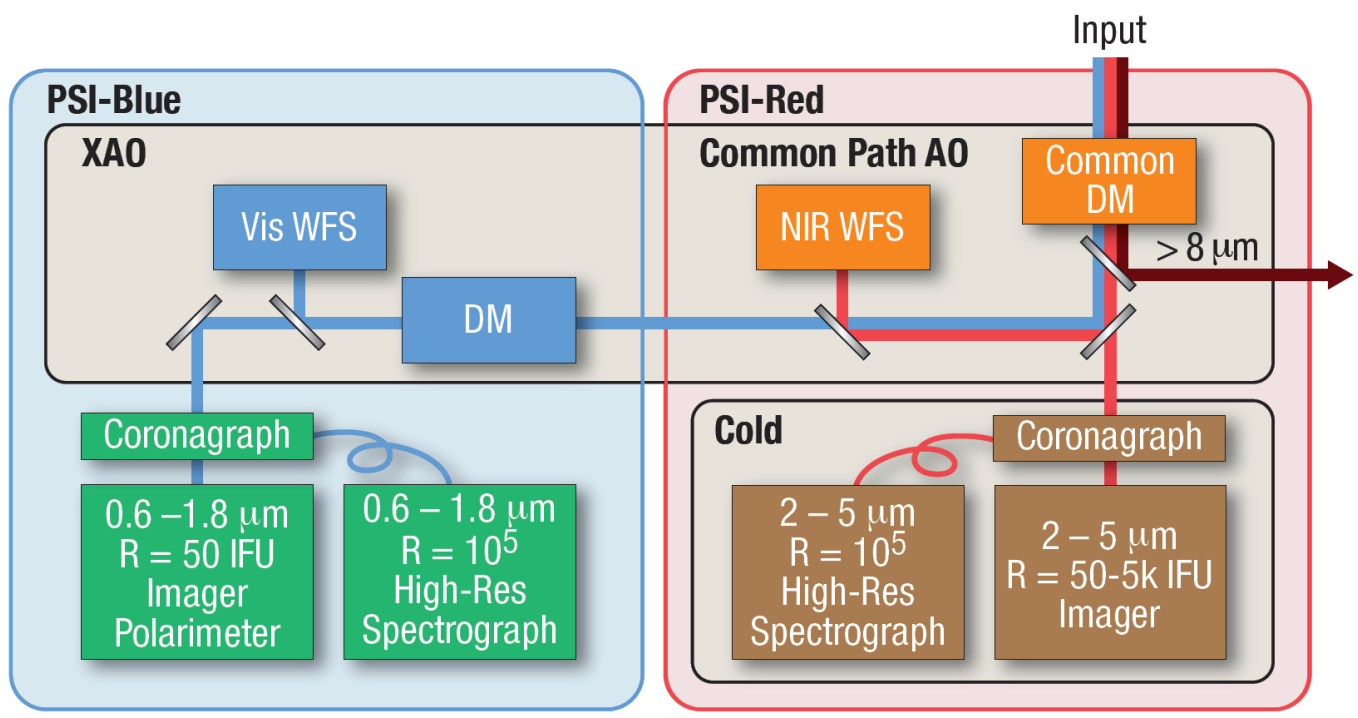

Figure 8. Configuration of PSI. 


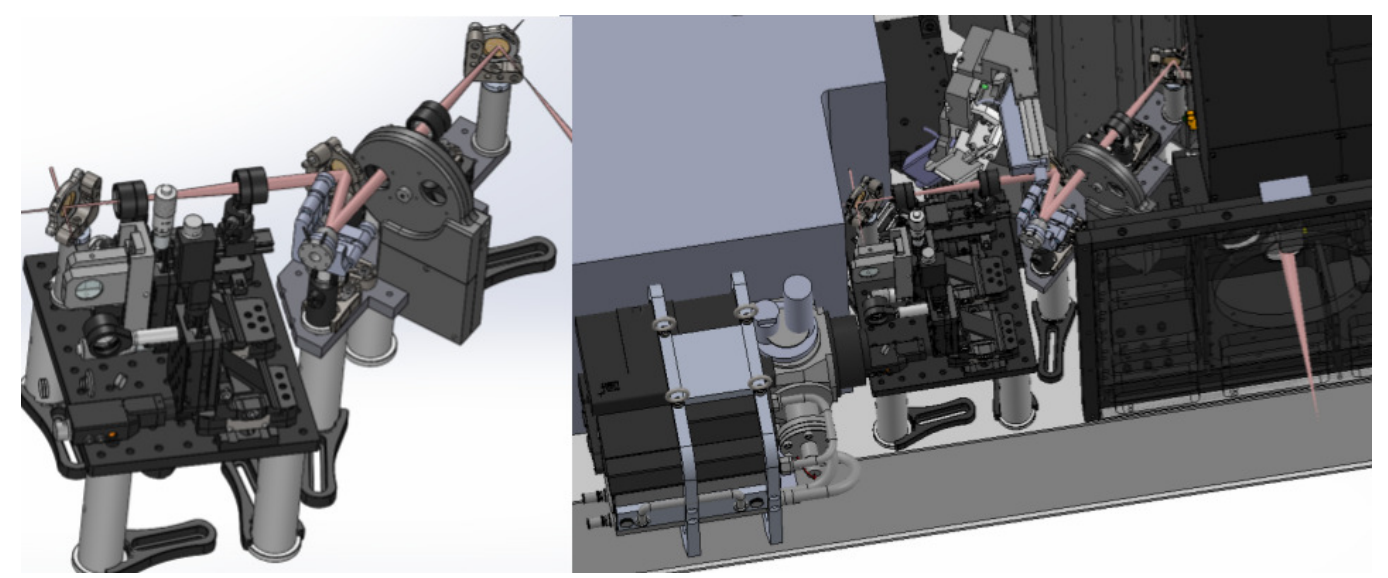

Figure 9. Current design of the NIR PyWFS for AO188.

The first planed upgrade is the addition of a NIR PyWFS inside AO188, using a First Light Imaging C-RED ONE camera (see Fig. 9). The camera was purchased partly using a grant which goal is to look at the galactic center with IRCS. But the PyWFS is design such as it can benefit both IRCS and SCExAO. It is designed using mostly off-the-shelf parts, with only a few custom optics and a custom pair of roof prisms similar to the ones used by SCExAO's visible PyWFS.

In 2021, AO188's DM will be replaced with ALPAO's 64x64 element DM, which is a half-scale version of the planned 128x128 DM used by PSI (Fig. 10 a)). This would increase the number of actuators in the pupil to more than 3000. The NIR PyWFS is currently designed with the DM upgrade in mind, such as when the pair is working together, we will achieve ExAO performances for IRCS and SCExAO, on redder stars such as M-type stars, directly after the first stage of correction.

Finally, in the next couple of years, a beam switcher will be added between AO188, SCExAO and IRCS (Fig. $10 \mathrm{~b}$ ) and c)). This beam switcher has several ports on all sides, that can accommodate up to 4 instruments at a time. The beam switcher is equipped with pickoff mirrors and dichroics that will distribute the light to the different ports. As represented on Fig. 10 b), SCExAO and the Infrared Camera and Spectrograph (IRCS) will occupy two of the ports, and can share the light using a $\sim 2 \mu \mathrm{m}$ dichroic. Although IRCS is not an IFS, this configuration will demonstrate an equivalent of the PSI-red+PSI-blue assembly. Logistically, the beam switcher will remove the necessity of craning either SCExAO or IRCS in front of AO188 depending on the observation, and even allow to observe in a mini queue mode depending on the atmospheric conditions.

In parallel to these major hardware changes, new advanced wavefront control algorithms such as coherent differential imaging, predictive control, sensor fusion or real-time post-processing. In addition to demonstrate
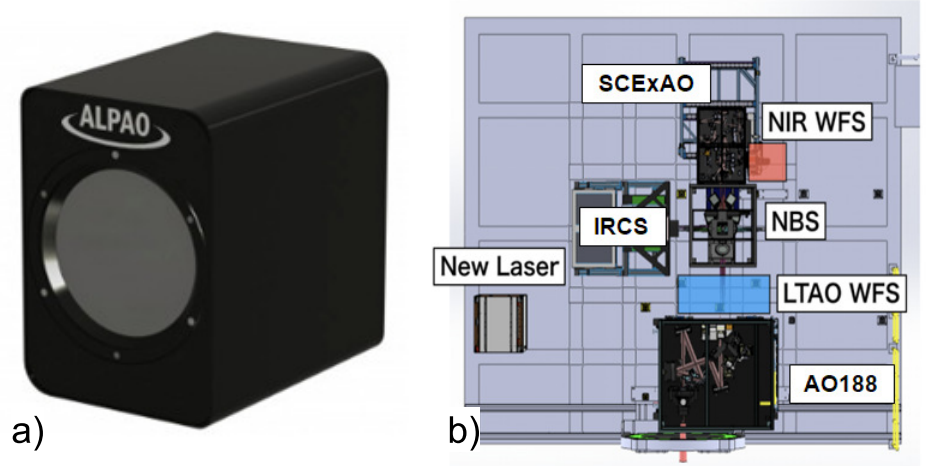

C)

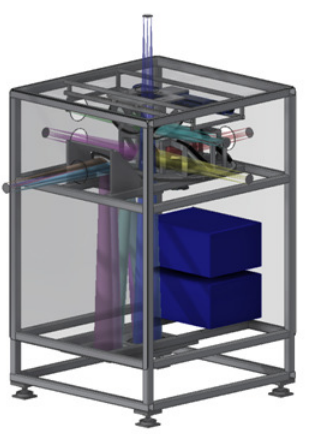

Figure 10. Hardware upgrades scheduled for AO188: a) new 64x64 ALPAO DM, b) Future configuration at the Nasmyth platform including a new beam switcher, allowing to split the light between SCExAO and IRCS, as well as other potential future instruments, c) new beam switcher with several ports on all sides. 
higher contrast in preparation for PSI, SCExAO will be able to image young Jupiter-mass planets closer to the habitable zone, down to $\sim 3 \mathrm{AU}$, where they should be more abundant. This will give us more insight on the planet population around the habitable zone. Finally, a few older Jupiter-size planets should be reached by looking at the reflected light for the first time.

\section{CONCLUSION}

Over the past 10 years, SCExAO has been evolving into a complex and modular instrument, containing all the components of classical high-contrast instrument, although with extra capabilities. This design allows for the team to collaborate with groups all over the world on innovative technologies and algorithms. The hardware and software architectures provide the necessary tools for collaborators to design, install and test their own project, in laboratory conditions and on-sky, with minimal effort for the SCExAO team. We are now testing new key technologies for future high-contrast imagers on GSMTs, such as the MKIDs detectors, or fast and low-noise IR detectors. One goal of SCExAO is to become a technology demonstrator and testbench for the future TMT high-contrast instrument PSI. In order to achieve this objective, major upgrade changes in SCExAO, but also in AO188 and the whole Nasmyth IR platform of Subaru will modify the instrument configuration to get closer to the combination PSI-red+blue. AO188 will receive a new $64 \times 64$ DM that will transform the first stage of turbulence correction into an ExAO system. Then a beam switcher and an IR PyWFS will complete the upgrades and provide more flexibility in the observing modes at Subaru. Once completed, the combination of SCExAO, AO188 and IRCS will be a fully complete system-level demonstrator for PSI.

\section{ACKNOWLEDGMENTS}

The development of SCExAO was supported by the Japan Society for the Promotion of Science (Grant-in-Aid for Research \#23340051, \#26220704, \#23103002, \#19H00703 \& \#19H00695), the Astrobiology Center of the National Institutes of Natural Sciences, Japan, the Mt Cuba Foundation and the director's contingency fund at Subaru Telescope. F. Martinache's work is supported by the ERC award CoG - 683029. The authors wish to recognize and acknowledge the very significant cultural role and reverence that the summit of Maunakea has always had within the indigenous Hawaiian community. We are most fortunate to have the opportunity to conduct observations from this mountain.

\section{REFERENCES}

[1] Jovanovic, N., Martinache, F., Guyon, O., Clergeon, C., Singh, G., Kudo, T., Garrel, V., Newman, K., Doughty, D., Lozi, J., Males, J., Minowa, Y., Hayano, Y., Takato, N., Morino, J., Kuhn, J., Serabyn, E., Norris, B., Tuthill, P., Schworer, G., Stewart, P., Close, L., Huby, E., Perrin, G., Lacour, S., Gauchet, L., Vievard, S., Murakami, N., Oshiyama, F., Baba, N., Matsuo, T., Nishikawa, J., Tamura, M., Lai, O., Marchis, F., Duchene, G., Kotani, T., and Woillez, J., "The Subaru Coronagraphic Extreme Adaptive Optics System: Enabling High-Contrast Imaging on Solar-System Scales," Pub. Astron. Soc. Pacific 127, 890 (Sep 2015).

[2] Fitzgerald, M., Bailey, V., Baranec, C., Batalha, N., Benneke, B., Beichman, C., Brandt, T., Chilcote, J., Chun, M., Crossfield, I., Currie, T., Davis, K., Dekany, R., Delorme, J.-R., Dong, R., Doyon, R., Dressing, C., Echeverri, D., Fortney, J., Frazin, R. A., Guyon, O., Hashimoto, J., Hillenbrand, L., Hinz, P., Howard, A., Jensen-Clem, R., Jovanovic, N., Kawahara, H., Knutson, H., Konopacky, Q., Kotani, T., Lafrenière, D., Liu, M., Lozi, J., Lu, J. R., Males, J., Marley, M., Marois, C., Mawet, D., Mazin, B., Millar-Blanchaer, M., Mondal, S., Murakami, N., Murray-Clay, R., Narita, N., Pezzato, J., Pyo, T.-S., Roberts, L., Ruane, G., Sallum, S., Serabyn, G., Shields, A., Simard, L., Skemer, A., Stelter, R. D., Tamura, M., Troy, M., Vasisht, G., Wallace, J. K., Wang, J., Wang, J., and Wright, S. A., "The Planetary Systems Imager for TMT," in [Bulletin of the American Astronomical Society], 51, 251 (Sep 2019).

[3] Sanders, G. H., "The Thirty Meter Telescope (TMT): An International Observatory," Journal of Astrophysics and Astronomy 34, 81-86 (June 2013). 
[4] Beuzit, J.-L., Feldt, M., Dohlen, K., Mouillet, D., Puget, P., Wildi, F., Abe, L., Antichi, J., Baruffolo, A., Baudoz, P., Boccaletti, A., Carbillet, M., Charton, J., Claudi, R., Downing, M., Fabron, C., Feautrier, P., Fedrigo, E., Fusco, T., Gach, J.-L., Gratton, R., Henning, T., Hubin, N., Joos, F., Kasper, M., Langlois, M., Lenzen, R., Moutou, C., Pavlov, A., Petit, C., Pragt, J., Rabou, P., Rigal, F., Roelfsema, R., Rousset, G., Saisse, M., Schmid, H.-M., Stadler, E., Thalmann, C., Turatto, M., Udry, S., Vakili, F., and Waters, R., "SPHERE: a 'Planet Finder' instrument for the VLT," in [Ground-based and Airborne Instrumentation for Astronomy II], Proc. Soc. Photo-Opt. Instrum. Eng. 7014, 701418 (July 2008).

[5] Macintosh, B., Graham, J. R., Ingraham, P., Konopacky, Q., Marois, C., Perrin, M., Poyneer, L., Bauman, B., Barman, T., Burrows, A. S., Cardwell, A., Chilcote, J., De Rosa, R. J., Dillon, D., Doyon, R., Dunn, J., Erikson, D., Fitzgerald, M. P., Gavel, D., Goodsell, S., Hartung, M., Hibon, P., Kalas, P., Larkin, J., Maire, J., Marchis, F., Marley, M. S., McBride, J., Millar-Blanchaer, M., Morzinski, K., Norton, A., Oppenheimer, B. R., Palmer, D., Patience, J., Pueyo, L., Rantakyro, F., Sadakuni, N., Saddlemyer, L., Savransky, D., Serio, A., Soummer, R., Sivaramakrishnan, A., Song, I., Thomas, S., Wallace, J. K., Wiktorowicz, S., and Wolff, S., "First light of the Gemini Planet Imager," Proceedings of the National Academy of Science 111, 12661-12666 (Sept. 2014).

[6] Minowa, Y., Hayano, Y., Oya, S., Watanabe, M., Hattori, M., Guyon, O., Egner, S., Saito, Y., Ito, M., Takami, H., Garrel, V., Colley, S., Golota, T., and Iye, M., "Performance of Subaru adaptive optics system AO188," in [Adaptive Optics Systems II], Proc. Soc. Photo-Opt. Instrum. Eng. 7736, 77363N (July 2010).

[7] Lozi, J., Jovanovic, N., Guyon, O., Chun, M., Jacobson, S., Goebel, S., and Martinache, F., "Visible and Near Infrared Laboratory Demonstration of a Simplified Pyramid Wavefront Sensor," Pub. Astron. Soc. Pacific 131, 044503 (Apr 2019).

[8] Singh, G., Lozi, J., Guyon, O., Baudoz, P., Jovanovic, N., Martinache, F., Kudo, T., Serabyn, E., and Kuhn, J., "On-Sky Demonstration of Low-Order Wavefront Sensing and Control with Focal Plane Phase Mask Coronagraphs," Pub. Astron. Soc. Pacific 127, 857-869 (Oct. 2015).

[9] Groff, T. D., Peters, M. A., Kasdin, N. J., Knapp, G., Galvin, M., Carr, M., McElwain, M. W., Brandt, T., Janson, M., Gunn, J. E., Lupton, R., Guyon, O., Martinache, F., Jovanovic, N., Hayashi, M., and Takato, N., "Design of the CHARIS integral field spectrograph for exoplanet imaging," in [Techniques and Instrumentation for Detection of Exoplanets VI], Proc. Soc. Photo-Opt. Instrum. Eng. 8864, 88640H (Sept. 2013).

[10] Currie, T., Brandt, T. D., Kuzuhara, M., Chilcote, J., Guyon, O., Marois, C., Groff, T., Lozi, J., Vievard, S., Sahoo, A., Deo, V., Jovanovic, N., Martinache, F., Wagner, K., Dupuy, T. J., Wahl, M., Letawsky, M., Li, Y., Zeng, Y., Brandt, G. M., Michalik, D., Grady, C., Janson, M., Knapp, G. R., Kwon, J., Lawson, K. D., McElwain, M., Uyama, T., Wisniewski, J. P., and Tamura, M., "Scexao/charis direct imaging discovery of a 20 au separation, low-mass ratio brown dwarf companion to an accelerating sun-like star," (2020).

[11] Currie, T., Marois, C., Cieza, L., Mulders, G. D., Lawson, K., Caceres, C., Rodriguez-Ruiz, D., Wisniewski, J., Guyon, O., Brandt, T. D., Kasdin, N. J., Groff, T. D., Lozi, J., Chilcote, J., Hodapp, K., Jovanovic, N., Martinache, F., Skaf, N., Lyra, W., Tamura, M., Asensio-Torres, R., Dong, R., Grady, C., Gerard, B., Fukagawa, M., Hand, D., Hayashi, M., Henning, T., Kudo, T., Kuzuhara, M., Kwon, J., McElwain, M. W., and Uyama, T., "No Clear, Direct Evidence for Multiple Protoplanets Orbiting LkCa 15: LkCa 15 bcd are Likely Inner Disk Signals," Astrophys. J. Lett. 877, L3 (May 2019).

[12] Hashimoto, J., Tamura, M., Muto, T., Kudo, T., Fukagawa, M., Fukue, T., Goto, M., Grady, C. A., Henning, T., Hodapp, K., Honda, M., Inutsuka, S., Kokubo, E., Knapp, G., McElwain, M. W., Momose, M., Ohashi, N., Okamoto, Y. K., Takami, M., Turner, E. L., Wisniewski, J., Janson, M., Abe, L., Brandner, W., Carson, J., Egner, S., Feldt, M., Golota, T., Guyon, O., Hayano, Y., Hayashi, M., Hayashi, S., Ishii, M., Kandori, R., Kusakabe, N., Matsuo, T., Mayama, S., Miyama, S., Morino, J. I., Moro-Martin, A., Nishimura, T., Pyo, T. S., Suto, H., Suzuki, R., Takato, N., Terada, H., Thalmann, C., Tomono, D., Watanabe, M., Yamada, T., Takami, H., and Usuda, T., "Direct Imaging of Fine Structures in Giant Planet-forming Regions of the Protoplanetary Disk Around AB Aurigae," Astrophys. J. Lett. 729, L17 (Mar 2011).

[13] Kotani, T., Tamura, M., Nishikawa, J., Ueda, A., Kuzuhara, M., Omiya, M., Hashimoto, J., Ishizuka, M., Hirano, T., Suto, H., Kurokawa, T., Kokubo, T., Mori, T., Tanaka, Y., Kashiwagi, K., Konishi, M., Kudo, T., Sato, B., Jacobson, S., Hodapp, K. W., Hall, D. B., Aoki, W., Usuda, T., Nishiyama, S., Nakajima, T., Ikeda, Y., Yamamuro, T., Morino, J.-I., Baba, H., Hosokawa, K., Ishikawa, H., Narita, N., Kokubo, E., 
Hayano, Y., Izumiura, H., Kambe, E., Kusakabe, N., Kwon, J., Ikoma, M., Hori, Y., Genda, H., Fukui, A., Fujii, Y., Kawahara, H., Olivier, G., Jovanovic, N., Harakawa, H., Hayashi, M., Hidai, M., Machida, M., Matsuo, T., Nagata, T., Ogihara, M., Takami, H., Takato, N., Terada, H., and Oh, D., "The infrared Doppler (IRD) instrument for the Subaru telescope: instrument description and commissioning results," in [Proc. Soc. Photo-Opt. Instrum. Eng.], Evans, C. J., Simard, L., and Takami, H., eds., Society of Photo-Optical Instrumentation Engineers (SPIE) Conference Series 10702, 1070211 (July 2018).

[14] Guyon, O., Sevin, A., Gratadour, D., Bernard, J., Ltaief, H., Sukkari, D., Cetre, S., Skaf, N., Lozi, J., Martinache, F., Clergeon, C., Norris, B., Wong, A., and Males, J., "The compute and control for adaptive optics (CACAO) real-time control software package," in [Proc. Soc. Photo-Opt. Instrum. Eng.], Society of Photo-Optical Instrumentation Engineers (SPIE) Conference Series 10703, 107031E (Jul 2018).

[15] Knight, J. M., Guyon, O., Lozi, J., Jovanovic, N., and Males, J. R., "Phase-induced amplitude apodization complex-mask coronagraph tolerancing and analysis," in [Proc. Soc. Photo-Opt. Instrum. Eng.], Society of Photo-Optical Instrumentation Engineers (SPIE) Conference Series 10706, $107065 \mathrm{O}$ (Jul 2018).

[16] Currie, T., Kasdin, N. J., Groff, T. D., Lozi, J., Jovanovic, N., Guyon, O., Brand t, T., Martinache, F., Chilcote, J., Skaf, N., Kuhn, J., Pathak, P., and Kudo, T., "Laboratory and On-sky Validation of the Shaped Pupil Coronagraph's Sensitivity to Low-order Aberrations With Active Wavefront Control," Pub. Astron. Soc. Pacific 130, 044505 (Apr 2018).

[17] Bos, S. P., Doelman, D. S., de Boer, J., Por, E. H., Norris, B., Escuti, M. J., and Snik, F., "Fully broadband vAPP coronagraphs enabling polarimetric high contrast imaging," in [Proc. Soc. Photo-Opt. Instrum. Eng.], Society of Photo-Optical Instrumentation Engineers (SPIE) Conference Series 10706, 107065M (Jul 2018).

[18] Sahoo, A., Guyon, O., Lozi, J., Chilcote, J., Jovanovic, N., Brandt, T., Groff, T., and Martinache, F., "Precision Photometric and Astrometric Calibration Using Alternating Satellite Speckles," aj 159, 250 (June 2020).

[19] Huby, E., Duchêne, G., Marchis, F., Lacour, S., Perrin, G., Kotani, T., Choquet, É., Gates, E. L., Lai, O., and Allard, F., "FIRST, a fibered aperture masking instrument. II. Spectroscopy of the Capella binary system at the diffraction limit," Astron. Astrophys. 560, A113 (Dec. 2013).

[20] Rains, A. D., Ireland, M. J., Jovanovic, N., Feger, T., Bento, J., Schwab, C., Coutts, D. W., Guyon, O., Arriola, A., and Gross, S., "Precision single mode fibre integral field spectroscopy with the RHEA spectrograph," in [Ground-based and Airborne Instrumentation for Astronomy VI], Proc. Soc. Photo-Opt. Instrum. Eng. 9908, 990876 (Aug. 2016).

[21] Bos, S. P., Vievard, S., Wilby, M. J., Snik, F., Lozi, J., Guyon, O., Norris, B. R. M., Jovanovic, N., Martinache, F., Sauvage, J. F., and Keller, C. U., "On-sky verification of Fast and Furious focal-plane wavefront sensing: Moving forward toward controlling the island effect at Subaru/SCExAO," aap 639, A52 (July 2020).

[22] N'Diaye, M., Martinache, F., Jovanovic, N., Lozi, J., Guyon, O., Norris, B., Ceau, A., and Mary, D., "Calibration of the island effect: Experimental validation of closed-loop focal plane wavefront control on Subaru/SCExAO," aap 610, A18 (Feb. 2018).

[23] Martinache, F., Guyon, O., Jovanovic, N., Clergeon, C., Singh, G., Kudo, T., Currie, T., Thalmann, C., McElwain, M., and Tamura, M., "On-Sky Speckle Nulling Demonstration at Small Angular Separation with SCExAO," Pub. Astron. Soc. Pacific 126, 565-572 (June 2014).

[24] Goebel, S. B., Hall, D. N. B., Guyon, O., Warmbier, E., and Jacobson, S. M., "Overview of the SAPHIRA detector for adaptive optics applications," Journal of Astronomical Telescopes, Instruments, and Systems 4, 026001 (Apr 2018).

[25] Miller, K., Guyon, O., and Males, J., "Spatial linear dark field control: stabilizing deep contrast for exoplanet imaging using bright speckles," Journal of Astronomical Telescopes, Instruments, and Systems 3, 049002 (Oct. 2017).

[26] Clergeon, C., Minowa, Y., Guyon, O., Ono, Y., Mieda, E., Skaf, N., Yoshida, H., Hayano, Y., Hattori, T., Schubert, K., Sahoo, A., Lozi, J., and Vievard, S., "Subaru AO188 upgrade phase 1: integration of the new real-time system," in [Proc. Soc. Photo-Opt. Instrum. Eng.], Society of Photo-Optical Instrumentation Engineers (SPIE) Conference Series 10703, 1070337 (Jul 2018). 